\title{
Specialized Vascular Access Teams
}

\author{
Peter J. Carr and Nancy L. Moureau
}

\section{Abstract}

Patients admitted to hospital are exposed to invasive procedures and will receive various interventions from different professional roles all with different levels of experience: some with limited experience and some with extensive experience. In simple terms, an orthopaedic surgeon fixes your broken bones, a cardiologist attends to your heart, a geriatrician focuses on elderly patient care, and a vascular surgeon focuses on vascular surgery. Yet, which vascular access device is inserted influences the clinical outcomes within each of these select specialties. Insertion procedures and care and maintenance are shared by a variety of healthcare professional disciplines, all with a variety of experience, guided by local policy frameworks. Because of this interdisciplinary sharing, responsibility becomes fragmented, and ownership of outcomes is lacking leading to increased patient safety risks. This chapter will

P. J. Carr $(\bowtie)$

HRB Clinical Research Facility Galway, National University of Ireland Galway,

University Hospital Galway, Galway, Ireland

e-mail: peter.carr@nuigalway.ie

\section{N. L. Moureau}

Menzies Health Institute, Alliance for Vascular Access Teaching and Research (AVATAR) Group, Griffith University, Brisbane, QLD, Australia e-mail: nancy@piccexcellence.com firstly identify the various definitions that make up a vascular access specialist team (VAST) and secondly the variety of evidence supporting the concept and what empirical guidelines say about it. Finally, it explores the use of a vascular access specialist teams to promote unity in patient care and assurance that only well-trained clinicians who are qualified to select, insert and care for VADs can do so, promoting greater patient safety and positive patient outcomes.

\section{Keywords}

Vascular access specialists · Vascular access teams $\cdot$ Benefits of VAST

\subsection{Introduction}

The scale of healthcare is arguably as big as any major industry with up to $85 \%$ of individuals worldwide seeking healthcare services once a year and a quarter of those exceeding four visits yearly (Rosen et al. 2018). Vascular access and intravenous therapy devices are a massive contributor to the invasive device market with annual worldwide use of VADs in the billions (Alexandrou et al. 2018). Yet the request, assessment and insertion of a VAD, the care and maintenance and, indeed, the removal of VADs are shared by a variety of healthcare professional disciplines.

When a patient is admitted to hospital, they are invariably exposed to a variety of invasive VAD procedures. Many will receive clinical interventions from 
different professional roles, all with different levels of experience following specific professional guidelines. Such a strategy is best represented as an interdisciplinary sharing method: one entity for insertion and another for care and maintenance. This process has resulted in responsibility for VADs being fragmented and this process identified as an increased patient safety risk (Castro-Sanchez et al. 2014). Specialist medical care is not so fragmented, for example, in specific healthcare domains of cardiology, orthopaedics, paediatrics and others all providing a higher level of quality care within their area of specialization. Therefore, it is important that highly skilled clinicians or specialists are available to improve VAD outcomes, ideally adopting a vessel health preservation (VHP) approach (Moureau and Carr 2018).

VAD practice is not yet globally accepted to be one that demands a specialist healthcare profession to ensure maximum patient safety with their use. It is often described as a generic skill or a clinical procedure absorbed into other competing clinical responsibilities and professional domains. Despite the pervasive use of VADs and associated technologies, the ownership of its term as a specialty or specialist team is in its infancy (Davis et al. 2016). This means high-level evidence for the specialty and teams is also lacking (Carr et al. 2018). Notwithstanding this reality, it is worth exploring the contribution of a vascular access specialist and team approach has made to patient's health and on the patient experience. Moreover, how a team approach can be adopted into a future where a safety science culture is likely to become commonly accepted in healthcare (Marshall et al. 2013) and where it can demonstrate sustained value as an authority on vascular access quality and safety (Dixon-Woods et al. 2013). The adoption and use of VHP and VAST will add important and timely insights into a concept eager for clinical validation.

\subsection{Vascular Access Teams Defined}

A vascular access team is the grouping of healthcare professionals whose primary role is to assess, insert, manage, perform surveillance, analyse their service data and solve clinical concerns and where possible remove VADs. The antecedents to a vascular access team were generalist nurses and medical doctors performing the insertion of VADs, be it peripheral or central catheters, with the latter overwhelmingly performed by medical doctors. However, the emergence of the peripherally inserted central catheter (PICC) has seen the adoption of PICC services, with the majority of PICC insertions led by the nursing workforce (Chopra et al. 2017a, b). Insertion of peripheral intravenous catheters (PIVC) has long been in the scope of nursing throughout most of the world, and this was recently validated (Alexandrou et al. 2018), and while physicians such as anaesthetists commonly place central venous catheters (CVC), nurses have increasingly assumed the role for PICCs and Midline insertions.

VADs are inserted under a plethora of health service models, medical only for central devices and nursing for other peripherally inserted devices; the adoption of a vascular access team could promote collegiate responsibility and lead to proactive decision-making, as opposed to developing a solution arising from an avoidable clinical problem. One recent example in the literature with regard to a difficult intravenous access patient cohort, identifies how a team approach can improve insertion outcomes in this specific patient population (Sou et al. 2017). An additional accepted term within a team approach includes the title vascular access specialist; referring to an individual clinician, (Marsh et al. 2018) with much variation in their description, professional role and clinical privileges. What is likely an encouraging phenomenon is that vascular access teams and specialists working within them appear to be initiated from a service context less clinical trial evidence. There is an opportunity for fuller scientific evaluation; both quality improvement and clinical research methods to evaluate the impact of the vascular access team concept. As a result a stronger empirically valid definitions of the team concept is likely to evolve. In future, the merging of healthcare and clinical disciplines such as surveillance scientists (Devries, 2016) within a team will signal a new era for vascular access science. Moreover, a patient included model of care that accepts the most appropriate device inserted and managed by a group of clinical experts analysing standardised data is likely to inform and provide the highest quality of care. 


\subsection{Vascular Access Specialist Defined}

Consensus regarding the term vascular access as a specialty is beginning to assume prominence in the literature (Davis et al. 2016), but it is fair to say it is a term that is not regularly used in hospitals worldwide. However, vascular access specialist is emerging as an accepted term in the literature (Chopra et al. 2017a, b). Adoption of this term and approach will likely occur, and one hopes it is introduced with appropriate evidence using appropriate methodologies. If we acknowledge and accept the necessity of VADs in current medical treatment, then by reason the use of vascular access specialists within a team will likely evolve into an established entity within the future of healthcare.

\subsection{Evidence Supporting Vascular Access Specialist Team}

In the 1980 the first controlled clinical trials investigating the impact of intravenous teams was reported with a primary outcome identifying the team approach reduced phlebitis (Tomford et al. 1984). Over a decade later, another clinical study asked the same question with similar results (Soifer et al. 1998). Both studies favoured the adoption and implementation of intravenous (IV) teams into clinical practice as a strategy to negative outcomes of IVs for reduced infection and phlebitis. In 2008 results from a dedicated IV team revealed a high percentage of sustained first-time insertion success (Carr et al. 2010).

Conceptually a vascular access specialist team (VAST) should be underpinned with the highest level of evidence (Carr et al. 2018). However, VAST can be accused of compromising VHP principles of right device selection in vascular access decision-making as some VASTs perform only PIVC insertion, some only PICCs and/or CVADs. Moreover, if a VAST approach focuses on an insertion-only model, it may limit the available time to follow up and troubleshoot VAD issues. Despite prospective studies revealing better insertion outcomes and reduced complications, many services require greater evidence to expand into a team approach and justify the economic benefit
(Bolton 2009; Hadaway et al. 2013). Furthermore, economically, the team approach appears to be more cost-effective (Robinson et al. 2005), as it is associated with greater first-time insertion success as well as reducing length of hospital stay, improving efficiency with supplies and reducing device complications. In addition, Cochrane reviews on the subject matter identify few controlled clinical trials on vascular access teams and specialists. Only one current trial on the World Health Organization's clinical trials registry was identified with one registered clinical trial awaiting publication (Carr et al. 2018).

As a result, uncertainties exist, and the efficacy or credibility of VASTs is limited to pre- and post-descriptive, observational and prospective studies on this subject. Moreover, when viewed individually, it is unclear from the limited, dated, underpowered randomized controlled trials (RCTs) available to date which vascular access insertion model is most effective. Specifically, in relation to RCTs, it is unclear if the specialist/ team is superior or equivalent to one with multiple clinicians inserting VADs. Table 5.1 displays a selection of benefits for healthcare facilities in adopting a VAST approach.

Table 5.1 Why healthcare facilities should adopt a VAST

\begin{tabular}{l} 
Centralizes an approach to the co-ordination of VAD \\
care \\
Create and sustain a consistent approach to VAD \\
underpinned by safety science \\
\hline Lead the implementation of multimodal initiatives to \\
improve VAD outcomes \\
\hline Interpret VAD data to monitor clinician performance \\
and policy compliance \\
\hline Establish VAST as the clinical lead and hospital \\
authority on VAD standards \\
\hline VAST will improved the patient experience \\
\hline VAST will use fewer VADs based on fewer attempts \\
\hline VAST will evaluate products and technology prior to \\
clinical adoption \\
\hline VAST would lead clinical simulation, education and \\
training of VAD \\
\hline VAST will promote patient education and ensure a \\
patient safety culture is maintained \\
\hline VAST is more likely to integrate visualization \\
technologies that promote greater insertion success \\
and reduce complications \\
\hline VAST can assist with implementation and translation \\
of new evidence
\end{tabular}




\subsection{What the Guidelines Recommend}

Scientific reports substantiate clinical guidelines and the variety published promote the concept of specialty training for healthcare professionals; the team approach and an improvement in the process of vascular access (Bodenham et al. 2016; Gorski et al. 2016; O'Grady et al. 2011; Royal College of Nursing 2016; Pittiruti and Scoppettuolo 2017). Regardless of the lack of high-quality interventional data to support strong recommendations for a VAST and the different terminology used to describe a team or group of specialist inserters, major clinical guidelines endorse their adoption where possible. Infusion Nursing Standards (INS) define an infusion team as one with a scope for clinical care that consists of a variety of activities related to the safe insertion, delivery and maintenance of all infusion and vascular access therapies including fluids and medications, blood and blood components and parenteral nutrition and acknowledge that dedicated infusion teams report better insertion success and reduce device complication (Gorski et al. 2016). INS adds that the VAST also provides a role for product evaluation and education resource, as well as a source to collect meaningful data (Gorski et al. 2016). Specifically, with regard to insertion, a European clinical manual for the insertion of PICCs and Midlines suggests that dedicated venous access teams lead to significant advantages, namely, safety, costeffectiveness and efficiency (Pittiruti and Scoppettuolo 2017). This group also suggested VAST collaboration with hospital pharmacy was important, thus highlighting another discipline involved in VHP. The Centers for Disease Control 2011 guidelines reference "IV Teams" and suggest the team approach leaves no doubt as to their effectiveness in reducing the incidence of CRBSI (O'Grady et al. 2011). However, there is also contradictory evidence in the CDC guideline that supports ward-based nurse to performing dressing changes, as opposed to a dedicated infusion team demonstrating a cost saving of $\$ 90,000$ per year (Abi-Said et al. 1999). A more recent guideline from the Association of Anaesthetists of
Great Britain and Ireland, titled Safe Vascular Access, recommend hospitals to develop a process to ensure patients receive effective, timely and safe vascular access (Bodenham et al. 2016). Conceivably, this could mean the development of specialist vascular access training and or a VAST approach adopting the principles of VHP (Hallam et al. 2016). Adopting such a strategy it appears would be necessary as the current systems for vascular access are variable. As a result, a lack of clinical consistency for patient outcomes occurs (Bodenham et al. 2016).

\subsection{Benefits of Vascular Access Specialist Team}

Identifying who is best qualified to insert and manage vascular devices could likely lead to adaption of better outcomes for patients, see Table 5.2. For example, difficult IV access and central venous catheter insertion are generally associated with surgeons, anaesthetics and intensivist, yet this group does not routinely perform CVC dressing changes. In following up the impact of initial insertion decisions on postinsertion complications, a better approach could reduce complications (Carr et al. 2017). Modification of insertion practice to promote dressing integrity could lead to a reduction of catheter-related infection as evidenced in a large intensive care study (Timsit et al. 2012).

Table 5.2 Patients benefit from a VAST

Patients will have their VAD assessment and insertion performed or proctored by a VAST experienced clinician

Patients will be able to enter a shared decision-making concept understanding alternative devices can be used ensuring the right device is used

The likelihood a VAD will be inserted on the first attempt is very high

Post-insertion complications will be reduced leading to uninterrupted therapy

Improved patient experience

Dedicated point of care resource to troubleshoot VAD issues or patient queries

Could be exposed to having better products and technology used to improve clinical outcomes 
Important publications, including one RCT, describe the effectiveness of nurses inserting acute CVCs and tunnelled devices; despite evidence of equivalent or better outcomes, they are also economically more efficient with high patient satisfaction scores (Boland et al. 2003; Hamilton 2005; Yacopetti et al. 2010).

Given the variety of disciplines and practices involved in the process required for VAD insertion and care, it's illogical for hospital not to incorporate a version of VAST. Expecting the current process of new clinicians to perform invasive procedures and without appropriate, consistent and recurrent training (Carr et al. 2011) or to accomplish a specific training scheme serves the clinician and educators not the patient (Moureau et al. 2013). In essence, patients want fewer attempts (Cooke et al. 2018). The best evidence to date is that insertion attempts are reduced with team or with specialist qualifications. Ensuring VASTs are better resourced and evolve to invite a wide variety of clinical disciplines and professional roles into it will surely strengthen its cause and will hopefully translate into stronger clinical outcomes and evidence.

\subsection{Summary}

VHP is a pathway that begins with assessment of patient veins and selection of the lowest-risk device to deliver the treatment plan. Integration of research and guidelines into VHP is designed to result in the best outcomes for patients, allowing completion of treatment with fewer VADs, less cost, fewer supplies and less time while limiting complications. With a thorough knowledge base of types of VADs available, criteria for use and training for insertion, clinicians are better able to meet the needs of patients in a safe and efficient manner. Specially trained clinicians with protected time and defined clinical roles working in a VAST are better equipped to provide consultation for appropriate device, maintain first-time success with device insertion and report the lowest risk of complications with invasive VAD procedures.

\section{Case Study}

A hospital in the inner city noted a steady increase in CLABSI. All CVADs were placed by doctors either surgeons or in radiology department. In addition, multiple PIVC-related infections were noted over the past 12 months. A committee was established to study the problem as well as complaints of excessive PIVC attempts, complications associated with PIVCs and long wait time for CVADs. The committee noted a high total purchase rate for PIVCs equating five catheters per patient admission for the year (catheters purchased/patients admitted $=$ catheters per patient admission). Supplies and cost per placement were evaluated to be approximately $\$ 24.65$ per PIVC attempt. PIVC failure rate was approximately 55\% within the first $48 \mathrm{~h}$. Observations identified fragmentation, inconsistency and frequent breaks in aseptic technique during PIVC and CVAD insertions. A determination was made to develop training and education for insertion, vein selection, appropriate use of supplies, Aseptic Non Touch Technique (ANTT) and care and maintenance with all VADs. Evaluation of published research pointed to added benefits of establishing specialists for insertion. A pilot study was created within a unit of the hospital with the highest complications. The unit was staffed with a trained vascular access specialist. After 3 months improvement was noted in patient satisfaction, reduced use of supplies and complication reduction by more than $65 \%$ in CVADs and $82 \%$ in PIVCs. Catheter usage for the study unit was reduced to less than two catheters per patient admission. PIVC failure rate was reduced to less than $20 \%$ in $72 \mathrm{~h}$. The data from the pilot study demonstrated significant cost saving calculated and presented to the Chief Financial Officer. Plans for expansion of the specialist model for other units were proposed and accepted by administration. 


\section{Summary of Key Points}

1. Patients have the right to the most qualified inserter for every insertion; hospitals should therefore consider vascular access teams.

2. In future, vascular access teams will likely evolve to include an assessment; insertion and post insertion clinical resource.

3. Respected clinical guidelines recommend the vascular access team/specialist approach despite limited empirical evidence.

4. There is an opportunity for the vascular access team to disrupt and become a new innovation in healthcare.

5. Vascular access teams are possibly the best advocate to translate and implement the VHP concept.

\section{References}

Abi-Said D, Raad I, Umphrey J, Gonzalez V, Richardson D, Marts K, Hohn D. Infusion therapy team and dressing changes of central venous catheters. Infect Control Hosp Epidemiol. 1999;20:101-5.

Alexandrou E, Ray-Barruel G, Carr PJ, Frost S, Inwood S, Higgins N, Lin F, Alberto L, Mermel L, Rickard CM. Use of short peripheral intravenous catheters: characteristics, management, and outcomes worldwide. J Hosp Med. 2018;13.

Bodenham A, Babu S, Bennett J, Binks R, Fee P, Fox B, Johnston A, Klein A, Langton J, Mclure H. Association of Anaesthetists of Great Britain and Ireland. Safe vascular access 2016. Anaesthesia. 2016;71:573-85.

Boland A, Haycox A, Bagust A, Fitzsimmons L. A randomised controlled trial to evaluate the clinical and cost-effectiveness of Hickman line insertions in adult cancer patients by nurses. Health Technol Assess. 2003;7(36):iii, ix-x, 1-99. NCCHTA.

Bolton D. Writing a business case for the expansion of service: expanding the IV therapy team, from start to finish. J Infect Prev. 2009;10:S27-32.

Carr PJ, Glynn RW, Dineen B, Kropmans TJ. A pilot intravenous cannulation team: an Irish perspective. $\mathrm{Br}$ J Nurs. 2010;19:S19-27.

Carr PJ, Glynn RW, Dineen B, Devitt D, Flaherty G, Kropmans TJ, Kerin M. Interns' attitudes to IV cannulation: a KAP study. Br J Nurs. 2011;20:S15-20.
Carr P, Higgins N, Cooke M, Mihala G, Rickard C. Vascular access specialist teams for device insertion and prevention of failure. Cochrane Database Syst Rev. 2014;2014.

Carr PJ, She GN, Pickersgill H, Bessley T, Murray K, Jackson G, Ho K, Litton E. The state of the art of central venous catheter care in the intensive care unit of a tertiary hospital; A quality initiative and call to action. Aust Crit Care. 2017;30:112.

Carr PJ, Higgins NS, Cooke ML, Mihala G, Rickard CM. Vascular access specialist teams for device insertion and prevention of failure. The Cochrane Library. 2018.

Castro-Sanchez E, Charani E, Drumright L, Sevdalis N, Shah N, Holmes A. Fragmentation of care threatens patient safety in peripheral vascular catheter management in acute care-a qualitative study. PLoS One. 2014;9:e86167.

Chopra V, Kuhn L, Ratz D, Shader S, Vaughn VM, Saint S, Krein SL. Vascular access specialist training, experience, and practice in the United States. J Infus Nurs. 2017a;40:15-25.

Chopra V, Kuhn L, Ratz D, Winter S, Carr PJ, Paje D, Krein SL. Variation in use of technology among vascular access specialists: an analysis of the PICC1 survey. J Vasc Access. 2017b;18:243-9.

Cooke M, Ullman AJ, Ray-Barruel G, Wallis M, Corley A, Rickard CM. Not "just" an intravenous line: consumer perspectives on peripheral intravenous cannulation (PIVC). An international cross-sectional survey of 25 countries. PLoS One. 2018;13:e0193436.

Davis L, Owens A, Thompson J. Defining the specialty of vascular access through consensus: shaping the future of vascular access. J Assoc Vasc Access. 2016;21:125-30.

DeVries M, Valentine M, Mancos P. Protected clinical indication of peripheral intravenous lines: successful implementation. J Assoc Vasc Access. 2016;21(2):89-92

Dixon-Woods M, Leslie M, Tarrant C, Bion J. Explaining Matching Michigan: an ethnographic study of a patient safety program. Implement Sci. 2013;8:70.

Gorski L, Hadaway L, Hagle M, Mcgoldrick M, Orr M, Doellman D. Infusion therapy: standards of practice. J Infus Nurs. 2016;39(Suppl 1):S1-S159.

Hadaway L, Dalton L, Mercanti-Erieg L. Infusion teams in acute care hospitals: call for a business approach: an infusion nurses society white paper. J Infus Nurs. 2013;36:356-60.

Hallam C, Weston V, Denton A, Hill S, Bodenham A, Dunn H, Jackson T. Development of the UK Vessel Health and Preservation (VHP) framework: a multi-organisational collaborative. J Infect Prev. 2016;17:65-72.

Hamilton H. A nurse-led central venous vascular access service in the United Kingdom. J Assoc Vasc Access. 2005;10:77-80.

Marsh N, Webster J, Larsen E, Genzel J, Cooke M, Mihala G, Cadigan S, Rickard CM. Expert versus generalist inserters for peripheral intravenous catheter insertion: a pilot randomised controlled trial. Trials. 2018;19(1). 
Marshall M, Pronovost P, Dixon-Woods M. Promotion of improvement as a science. Lancet. 2013;381:419-21.

Moureau NL, Carr PJ. Vessel Health and Preservation: a model and clinical pathway for using vascular access devices. Br J Nurs. 2018;27:S28-35.

Moureau N, Lamperti M, Kelly L, Dawson R, Elbarbary M, Van Boxtel J, Pittiruti M. Evidence-based consensus on the insertion of central venous access devices: definition of minimal requirements for training. Br J Anaesth. 2013;110:333-46.

O'Grady N, Alexander M, Burns L, Dellinger E, Garland J, Heard S, Lipsett P, Masur H, Mermel L, Pearson M, Raad I, Randolph A, Rupp M, Saint S, Healthcare Infection Control Practices Advisory Committee (HICPAC). Centers for Disease Control Guidelines for the prevention of intravascular catheter-related infections. Clin Infect Dis. 2011;52:e162-93.

Pittiruti M, Scoppettuolo G. Gavecelt manual of PICC and midline: indications, insertion, management, Italy. Edna S.p.A.; 2017.

Robinson M, Mogensen K, Grudinskas G, Kohler S, Jacobs D. Improved care and reduced costs for patients requiring peripherally inserted central catheters: the role of bedside ultrasound and a dedicated team. J Parenter Enter Nutr. 2005;29:374-9.

Rosen MA, Diazgranados D, Dietz AS, Benishek LE, Thompson D, Pronovost PJ, Weaver SJ. Teamwork in healthcare: key discoveries enabling safer, highquality care. Am Psychol. 2018;73:433.

Royal College of Nursing. Standards for infusion therapy. In: Denton A, editor. . London: Royal College of Nursing; 2016.

Soifer N, Borzak S, Edlin B, Weinstein R. Prevention of peripheral venous catheter complications with an intravenous therapy team: a randomized controlled trial. Arch Intern Med. 1998;158:473-7.

Sou V, Mcmanus C, Mifflin N, Frost SA, Ale J, Alexandrou E. A clinical pathway for the management of difficult venous access. BMC Nurs. 2017;16:64.

Timsit J, Bouadma L, Ruckly S, Schwebel C, GarrousteOrgeas M, Bronchard R, Calvino-Gunther S, Laupland $\mathrm{K}$, Adrie $\mathrm{C}$, Thuong M. Dressing disruption is a major factor for catheter-related infections. Crit Care Med. 2012;40:1707-14.

Tomford JW, Hershey CO, Mclaren CE, Porter DK, Cohen DI. Intravenous therapy team and peripheral venous catheter-associated complications: a prospective controlled study. Arch Intern Med. 1984;144:1191-4.

Yacopetti N, Alexandrou E, Spencer T, Frost S, Davidson P, O'sullivan G, Hillman K. Central venous catheter insertion by a clinical nurse consultant or anaesthetic medical staff: a single-centre observational study. Crit Care Resusc. 2010;12:90-5.

Open Access This chapter is licensed under the terms of the Creative Commons Attribution 4.0 International License (http://creativecommons.org/licenses/by/4.0/), which permits use, sharing, adaptation, distribution and reproduction in any medium or format, as long as you give appropriate credit to the original author(s) and the source, provide a link to the Creative Commons license and indicate if changes were made.

The images or other third party material in this chapter are included in the chapter's Creative Commons license, unless indicated otherwise in a credit line to the material. If material is not included in the chapter's Creative Commons license and your intended use is not permitted by statutory regulation or exceeds the permitted use, you will need to obtain permission directly from the copyright holder. 